\title{
Multivectorial Strategy to Interpret a Resistive Behaviour of Loads in Smart Buildings
}

\author{
Manuel V. Castilla \\ Dept. Engineering Design \\ University of Seville \\ Seville, Spain \\ mviggo@us.es
}

\author{
Juan C. Bravo \\ Dept. Electrical Engineering \\ University of Seville \\ Seville, Spain \\ carlos_bravo@us.es
}

\author{
Francisco I. Martin \\ Dept. Electrical Engineering \\ University of Malaga \\ Malaga, Spain \\ fimartin@uma.es
}

\begin{abstract}
In Smart buildings, electric loads are affected by an important distortion in the current and voltage waveforms, caused by the increasing proliferation of non linear electronic devices. This paper presents an approach on non sinusoidal power theory based on Geometric Algebra that clearly improves traditional methods in the optimization of apparent power and power factor compensation. An example is included that demonstrates the superiority of this approach compared with traditional methods.
\end{abstract}

Keywords- building loads; geometric algebra (GA); multivector; resistive loads.

\section{INTRODUCTION}

Majority of harmonic problems affecting smart and industrial constructions are generated within the construction. This is due in part to a proliferation of non-linear loads connected to the circuits in the building. Air conditioning equipment, computers, fax and copying equipment, printers, TVs, adjustable speed drive (ASD), and other power electronic equipment, are the main sources of such problems. The result of using such highly non-linear load is that the current waveform is distorted, causing excessive harmonic of current and voltage. Also, the close proximity of many of these commercial buildings (hotels, offices, departmental stores, shopping centers, and hospitals) will definitely contribute to the distortion of the electric power quality of feeder, which supplies these buildings. These harmonics can cause serious problems in power systems, excessive heat of appliances, components aging and capacity decrease, fault of protection and measurement devices, lower power factor and consequently reducing power system efficiency due to increasing losses are some main effects of harmonics in power distribution systems. Harmonic distortion can cause significant costs in distribution networks. Harmonic cost consists of harmonic energy losses, premature aging and de-rating of electrical equipment. The difference between the known generation and the estimated consumption is considered as the energy loss. The harmful and cost effects of harmonics have been discussed extensively and spurred stringent requirements by international institutions regarding the allowed levels of harmonics at the point of connection to the power supply $[1,2]$.
In this sense, two compensator devices to power factor improvement are available in the case of smart buildings:

- Passive circuits with energy storing components.

- Active filters.

This work focuses mainly on passive circuits with energy storage elements. In this way, the objective function to minimize is a component of the apparent power named reactive power in different classical approaches.

At the end of $19^{\text {th }}$ century Steinmetz introduced the wellknown concept of Complex Power in a single-phase sinusoidal ac operation [3]. In the 1930's, Budeanu [4] proposed a certain decomposition of the Apparent Power in $n$-sinusoidal situations and Fryze [5] addressed the problem of the difference between the Apparent Power $S$ and the Active Power $P$ in the time domain. Both approaches were criticized much later by Czarnecki [6], who suggested reject the Budeanu reactive power component [7]. Triggered by these papers many investigations appeared consequently [8-13], each shedding more light on power factor improvement. In [7] a "reactive power" was defined that permits the derivation of the value of passive linear devices to achieve a relative optimum power factor. However, as Sharon pointed out [10], this reactive power is not minimized in general by this compensation.

Significant progress in the analysis of power equations has been made recently through the use of vector spaces [12]. Reference [14] introduces a new mathematical structure for a clear definition of vector-space applied to the power multivector concept. This quantity compound is thereby obtained involving complex scalar and complex bivector in the frequency domain.

In this paper, the power multivector concept defined in GA framework is used to probe that $P F$ is improved if and only if the electric circuit is dissipative. The dissipasitivity condition [17] is understood in terms of the available stored energy in the system. Consequently, the maximum power is consumed when the load is equivalent to a linear resistance. 


\section{REPRESENTATION ON PERIODIC SIGNALS}

Let us consider by considering a periodic waveform, $x(t)$, that can be expressed as an element of a function space

$L_{2}[0, T):=\left\{x(t):[0, T) \rightarrow \mathbb{R}^{n}:\|x(t)\|=\sqrt{\frac{1}{T} \int_{0}^{T} x^{2}(t) d t}\right.$

where the norm is defined as the rms value $\|x(t)\|$. The function space is mapped to a vector space $\mathcal{C G}_{n}$ [16] by representing the periodic waveforms as a linear combination of $n$ orthonormal basis vectors $\left\{\sigma_{1}, \sigma_{2}, \sigma_{3} \ldots \sigma_{n}\right\}$ [20]. The waveform $x(t)$ can be expressed as a linear combination of $n$ orthonormal basis functions $\varphi_{p}(t) \forall p=1 \ldots n$.

$$
x(t)=\sum_{p=1}^{n} x_{p} \varphi_{p}(t)
$$

and represented by a complex-vector (geometric phasor) in $\mathcal{C G}_{n}$ vector space

$$
\tilde{X}=\sum_{p=1}^{n} \bar{x}_{p} \sigma_{p}
$$

Due to the orthonormal character of the basis functions (2) may be written

$$
\|x(t)\|=\sqrt{\sum_{p=1}^{n} x_{p}^{2}}=\|\tilde{X}\|
$$

Note that in (4), the magnitude $\|\tilde{X}\|$ of the geometric phasor coincides with $\|x(t)\|$ rms value.

\section{POWER DECOMPosition}

In order to better understand the differences of classical power approaches respect to the power multivector on power factor compensation, it is briefly illustrated on Budeanu's power [4] versus power multivector [16]. This original quantity condenses all the information needed to solve future problems on power theory and plays a similar role to the Steinmetz model in the sinusoidal case.

\section{A. Classical Approach}

Budeanu suggested that the apparent power consists of three components, active power $\mathrm{P}$, reactive power $\mathrm{Q}$ and distortion power D. Budeanu's power equation is given by

$$
S_{B}^{2}=P_{B}^{2}+Q_{B}^{2}+D_{B}^{2}
$$

where,

$$
\begin{aligned}
& \quad P_{B}=\sum_{P} U_{p} I_{p} \cos \varphi_{p}, \quad Q_{B}=\sum_{p} U_{p} I_{p} \sin \varphi_{p} \quad \text { and } \\
& D_{B}=\sqrt{S^{2}-P^{2}-Q^{2}}
\end{aligned}
$$

\section{B. Multivectorial Approach}

For clarity of presentation and without loss of generality, the phase angles of the harmonic voltages are $\alpha_{p}=0$ and the load is linear. In this case, the power multivector $\tilde{S}$ entering the one-port in Fig. 1, is given by the following set

$$
\tilde{S}=\tilde{U} \odot \tilde{I}^{*}=\{\underbrace{\tilde{U} \cdot \tilde{I}^{*}}_{\tilde{\Omega}^{\bullet}} \oplus \underbrace{\tilde{U} \wedge \tilde{I}^{*}}_{\tilde{\Omega}^{\wedge}}\}
$$

which fits the energy conservation law [18].

In Fig. 1, the voltage and current of the ideal source are time functions and are denoted by $u(t), i(t) \in \mathbb{R}^{n}$ and $u(t), i(t) \in \mathcal{V}^{n} \in L_{2}^{n}[0, T]$. It is assumed that both quantities admit a geometric phasor representation, $\tilde{U}$ and $\tilde{I}$ respectively. Moreover, the set $\mathcal{V}^{n}$ may be equal to $L_{2}^{n}[0, T]$, a periodic signal or a set of sinusoids with limited content harmonic. The load is described by a linear/nonlinear circuit, Fig. 1. This figure shows the circuit of a diode rectifier with a capacitive output filter, which is an example of a nonlinear load with harmonic voltage source behaviour. This kind of circuit is present in almost all loads in smart buildings, such as new electronic devices, $\mathrm{AC} / \mathrm{DC}$ converters, electronic lamp ballasts, etc.

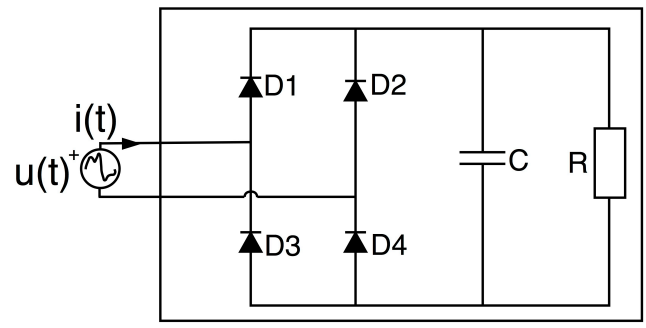

Fig. 1. Linear/Non-linear Load.

In this case, the presence of distorted current $i(t)$ has the deleterious effect of reducing the power transmission efficiency. The expression of voltage and current geometric phasors may be written as

$$
\begin{gathered}
\tilde{U}=\sum_{p \in N}\left\|\tilde{U}_{p}\right\| e^{j 0} \sigma_{p} \\
\tilde{I}=\sum_{q \in N}\left\|I_{q}\right\| e^{j \varphi_{q}} \sigma_{q}
\end{gathered}
$$

The harmonic current geometric phasor $\tilde{I}_{q}$ can be splitted as follows

$$
\tilde{I}_{q}=\bar{I}_{q} e^{-j \varphi_{q}} \sigma_{q}=\bar{I}_{q} \cos \varphi_{q} \sigma_{q}-j \bar{I}_{q} \sin \varphi_{q} \sigma_{q}=\tilde{I}_{q \|}-j \tilde{I}_{q \perp}
$$

where the subscripts " $\| "$ and " $\perp$ " indicate "in phase" and "in quadrature" respectively. Linear conjugate harmonic current $\tilde{I}_{q}^{*}$ is given by 


$$
\tilde{I}_{q}^{*}=\tilde{I}_{q \|}^{*}+j \tilde{I}_{q \perp}^{*}
$$

where $(*)$ is the standard "conjugate complex" operation.

In the expanded form of (6), the two components $\tilde{\Omega}^{\bullet}$ (scalar complex) and $\tilde{\Omega}^{\wedge}$ (bivector complex) can be expressed as

$$
\begin{aligned}
& \tilde{\Omega}^{\bullet}=\sum_{P}\left(U_{p} I_{p} \cos \varphi_{p}+j U_{p} I_{p} \sin \varphi_{p}\right) \sigma_{0} \\
& \tilde{\Omega}^{\wedge}=\sum_{\substack{p<q \\
p, q \in N}}\left\{\left(U_{p} I_{q} e^{j \varphi_{q}}-U_{q} I_{p} e^{j \varphi_{p}}\right) \sigma_{p q}\right\}
\end{aligned}
$$

\section{A Multivectorial Characterization Of Power FACTOR IMPROVEMENT}

It is addressed how the superiority of multivectorial representation of the reactive current and power components can be particularly useful compared to any classical definition.

Consequently, the PF is improved if and only if the compensated electric circuit is resistive. Thus, the maximum power is consumed when the load is equivalent to a linear resistance. The power factor compensation architecture is represented in Fig.2, where

$$
B_{c}(j \omega), Y_{l}(j \omega): \mathcal{V}^{n} \rightarrow L_{2}^{n}[0, T]
$$

are the susceptances of the compensator and the admitances of load, respectively.

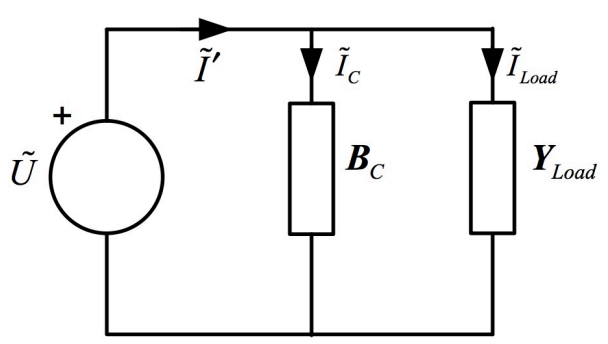

Fig. 2. Linear/Non-linear load with n-sinusoidal waveforms represented by its susceptances $B_{c}$, is placed in shunt.

Therefore, the power factor is improved if the following condition is fulfilled

$$
\int_{0}^{T} \xi\left(\tilde{u}, \tilde{i^{\prime}}\right) d t>0 \quad \forall\left(\tilde{u}, \tilde{i^{\prime}}\right) \in \mathcal{V}^{n} \times L_{2}^{n}[0, T]
$$

where $\tilde{u}=\Gamma_{F}(\tilde{U}), \tilde{i}^{\prime}=\Gamma_{F}\left(\tilde{I}^{\prime}\right), \Gamma_{F}$ is the Clifford-Fourier transform [19] and Appendix E in [16], and $\tilde{U}$ and $\tilde{I}^{\prime}$ are the geometric phasor of voltage and current. On the other hand, the function $\xi\left(\tilde{u}, \tilde{i}^{\prime}\right)$ in (13) is given by

$$
\begin{gathered}
\xi\left(\tilde{u}, \tilde{i}^{\prime}\right)=\xi\left(\sum_{p}\left|\tilde{U}_{p}\right| e^{j\left(p \omega t+\alpha_{p}\right)} \sigma_{p}, \sum_{q}\left|\tilde{I}_{q}^{\prime}\right| e^{j\left(q \omega t+\beta_{q}\right)} \sigma_{q}\right)= \\
=\left[Y_{\text {load }} \tilde{u}+\tilde{i}^{\prime}\right]^{T}\left[Y_{\text {load }} \tilde{u}-\tilde{i}^{\prime}\right]
\end{gathered}
$$

Consequently,

$$
B_{c}: \tilde{U} \rightarrow \tilde{I}_{c} \quad Y_{\text {load }}: \tilde{U} \rightarrow \tilde{I}_{\text {load }}
$$

where, $\tilde{I}_{c}, \tilde{I}_{l}$ denote the geometric phasor of compensator and load currents respectively.

From Kirchhoff's node law in Fig.2, $i^{\prime}=\tilde{i}_{C}+\tilde{i}_{L}$ load , where $\tilde{i}^{\prime}$ is the instantaneous current after compensation, and the following equation

$$
\tilde{i}_{C}=B_{C} \tilde{u}
$$

it follows that

$$
\left\langle\tilde{u}, B_{C} \tilde{u}\right\rangle=0
$$

resulting

$$
\left\langle\tilde{u}, \tilde{i}^{\prime}\right\rangle=\left\langle\tilde{u}, \tilde{i}_{\text {load }}\right\rangle
$$

Thus, the power factor PF is given by

$$
P F=\frac{\left\langle\tilde{u}, \tilde{i}_{\text {load }}\right\rangle}{\|\tilde{u}\| \tilde{i}^{\prime} \|}=\frac{\left\|\sum_{p} \tilde{U}_{p} \tilde{I}_{p_{\text {lood }}} \cos \varphi_{p}\right\|}{\|\tilde{u}\| \tilde{i}^{\prime} \|}
$$

Therefore, $B_{C}$ improves the $P F$ for a $Y_{l o a d}$ given if and only if $B_{C}$ satisfies

$$
2\left\langle Y_{\text {load }} \tilde{u}, B_{c} \tilde{u}\right\rangle+\left\|B_{c} \tilde{u}\right\|^{2}<0 \quad \forall \tilde{u} \in \mathcal{V}^{n}
$$

Equation (20) implies that

$$
\left\|\tilde{i}^{\prime}\right\|^{2}<\left\|Y_{\text {load }} \tilde{u}\right\|^{2}=\left\|\tilde{i}_{\text {load }}\right\|^{2}
$$

Clearly on can express the equation (16) as

$$
\begin{aligned}
& \int_{0}^{T} {\left[Y_{\text {load }} \tilde{u}+\tilde{i}^{\prime}\right]^{T}\left[Y_{\text {load }} \tilde{u}-\tilde{i^{\prime}}\right] d t=} \\
& \quad=\int_{0}^{T}\left\{\left\|Y_{\text {load }} \tilde{u}\right\|^{2}-\left\|\tilde{i}^{\prime}\right\|^{2}\right\} d t>0
\end{aligned}
$$

Equation (13) is equivalent to (22) and the susceptances $B_{C}$ improves the PF if and only if $B_{C}$ satisfies (20). Then, the current multivector $\tilde{I}$ in Fig. 1 is modified into $\tilde{I}^{\prime}$ in Fig. 2. Hence, the reactive current multivector $\left\|\sum_{q} \tilde{i}_{q \perp}\right\|$ is modified into 
$\left\|\sum_{q} \tilde{i}_{q \perp}^{\prime}\right\|$ and the "reactive power" multivector $\operatorname{Im} \tilde{\Omega}^{\bullet}=\tilde{Q}$ is modified into $\left(\operatorname{Im} \tilde{\Omega}^{\bullet}\right)^{\prime}=\tilde{Q}^{\prime}$ multivector and power bivector $\tilde{\Omega}^{\wedge}$ is modified into $\left(\tilde{\Omega}^{\wedge}\right)^{\prime}$. In this sense, $(21)$ is also given by

$$
\left\|\sum_{q} \tilde{i}_{q \perp}^{\prime}\right\|^{2} \leq\left\|Y_{\text {load }} \tilde{u}\right\|^{2}
$$

where $\sum_{q} \tilde{i}_{q \perp}^{\prime}$ is a component term of $\tilde{i}^{\prime}$.

\section{A hipothetical Case Study}

The main contribution of this paper is the identification of the role played by the GA to interpret the resistive behaviour for power factor compensation. To illustrate this, a theoretical case study is developed where the limitations of any reactive power component by itself are manifest. Units of physical quantities are those standards for the MKSA system and thus can be omitted.

Consider an elementary building circuit, constituted by a linear load supplied from a non-sinusoidal source.

$$
u(t)=\sqrt{2}[200 \sin \omega t+200 \sin (3 \omega t)]
$$

with $\omega=1 \mathrm{rad} / \mathrm{s}$.

The load impedance at fundamental frequency is $Z_{1}=1-3 j$. For the third harmonic they become $Z_{3}=1+j$. The voltage for the source and the current geometric phasor in connection with (7) and (8) are

$$
\begin{gathered}
\tilde{U}=200 \sigma_{1}+200 \sigma_{3} \Rightarrow\|\tilde{U}\|=282.84 \\
\tilde{I}=(20+j 60) \sigma_{1}+(100-j 100) \sigma_{3} \Rightarrow \| \tilde{I}||=154.9
\end{gathered}
$$

From (9), the corresponding "in quadrature" current is

$$
j \tilde{I}_{\perp}=j\left(60 \sigma_{1}-100 \sigma_{3}\right)
$$

From (5) and (11), the apparent power multivector and the apparent power Budeanu's are respectively

$$
\begin{gathered}
\tilde{S}=\left[(24+j 8) \sigma_{0}+(16+j 32) \sigma_{13}\right] 10^{3} \\
S_{B}=\|\tilde{S}\|=43.82 \cdot 10^{3} \Rightarrow P F=0.55 \\
\tilde{P}=\operatorname{Re}\left\{\Omega^{\bullet}\right\}=24 \times 10^{3} \omega_{0} \\
P_{B}=\|\tilde{P}\|=24 \times 10^{3} \\
\tilde{Q}=\operatorname{Im}\left\{\Omega^{\bullet}\right\}=j 8 \cdot 10^{3} \sigma_{0} \\
Q_{B}=\|\tilde{Q}\|=8 \cdot 10^{3}
\end{gathered}
$$

and

$$
\begin{aligned}
& \tilde{\Omega}^{\wedge}=(16+j 32) \sigma_{13} \\
& D_{B}=\left\|\tilde{\Omega}^{\wedge}\right\|=35.78
\end{aligned}
$$

TABLE I. POWER BEFORE TO COMPENSANTION

\begin{tabular}{|l|l|l|}
\hline \multicolumn{1}{|c|}{ Multivectorial Approach } & Classical Approach & Power factor \\
\cline { 1 - 2 }$\tilde{\mathrm{P}}=24 \cdot 10^{3} \sigma_{0}$ & $P_{B}=24 \cdot 10^{3}$ & \multirow{2}{*}{$P F=\frac{\|\tilde{P}\|}{\|\tilde{S}\|}=0.55$} \\
\cline { 1 - 2 }$=j 8 \cdot 10^{3} \sigma_{0}$ & $Q_{B}=8 \cdot 10^{3}$ & \\
\cline { 1 - 2 }$\tilde{\Omega}^{\wedge}=(16+j 32) \cdot 10^{3} \sigma_{13}$ & $D_{B}=35.78$ & \\
\cline { 1 - 2 }$=\left[\begin{array}{l}(24+j 8) \sigma_{0}+ \\
(16+j 32) \sigma_{13}\end{array}\right] 10^{3}$ & $S_{B}=43.82 \cdot 10^{3}$ & \\
\hline
\end{tabular}

With regard to the power factor improvement, let consider that a capacitive susceptance $B_{C}$ is connected to the load

\begin{tabular}{|c|c|c|}
\hline $\begin{array}{l}\text { Multivectorial } \\
\text { approach }\end{array}$ & Classical approach & Power factor \\
\hline$\tilde{P}=24 \cdot 10^{3} \sigma_{0}$ & $P_{B}=24 \cdot 10^{3}$ & \multirow{4}{*}{$P F=\frac{\|\tilde{P}\|}{\|\tilde{S}\|}=0.63$} \\
\hline$\tilde{Q}=-j 11.2 \cdot 10^{3} \sigma_{0}$ & $Q_{B}=11.2 \cdot 10^{3}$ & \\
\hline$\tilde{\Omega}^{\wedge}=(16+j 22.4) \cdot 10^{3} \sigma_{13}$ & $D_{B}=27.53 \cdot 10^{3}$ & \\
\hline$\tilde{S}=\left[\begin{array}{l}(24-j 11.2) \sigma_{0}+ \\
+(16+j 22.4) \sigma_{13}\end{array}\right] \cdot 10^{3}$ & $S_{B}=38.2 \cdot 10^{3}$ & \\
\hline
\end{tabular}
terminals of the elementary building circuit in Fig. 3. The current geometric phasor through the capacitor is given by

$$
j \tilde{I}_{C}=j\left(200 C \sigma_{1}+600 C \sigma_{3}\right)
$$

The quadrature current geometric phasor after compensation in circuit $a$ is now

$$
\tilde{I}_{\perp}^{\prime}=\tilde{I}_{\perp}+\tilde{I}_{C}=\left\{\begin{array}{l}
{[(60+200 C)] \sigma_{1}+} \\
{[(-100+600 C)] \sigma_{3}}
\end{array}\right\}
$$

in which $\left\|\tilde{I}_{\perp}^{C}\right\|$ is minimized when $C=0.12 F$. Then, the power factor is now $P F=0.63$. The results after capacitive compensation are summarized in Table II.

TABLE II. POWER AFTER CAPACITIVE COMPENSATION

\section{CONCLUDING REMARKS AND FUTURE RESEARCH}

This paper advances an analysis and compensator design for power factor improvement based on dissipativity condition derived from the geometric algebra framework. The more relevant comments resulting from a comparative analysis of these results and the classical approach are: 
- Classical apparent power (5) is only a secondary result of the suggested multivector (6). The apparent power $S_{B}$ is derived as the magnitude of the multivector $\tilde{S}$ and not as the formal product of the rms values of voltage and current waveforms.

- It is clear in Section III that

$$
\left\|\tilde{i}^{\prime}\right\|^{2}=\left\|\tilde{i}_{c}\right\|^{2}+\left\|\tilde{i}_{\text {load }}\right\|^{2}+2\left\langle\tilde{i}_{c}, \tilde{i}_{\text {load }}\right\rangle
$$

is a necessary and sufficient condition for minimizing $\left\|\sum_{q} \tilde{i}_{q \perp}\right\|$, as in (20), which is equivalent to power factor improvement as shown in (15).

- The numerical example is analysed using information exclusively contained in the power multivector.

- Although $S_{B}=\|\tilde{S}\|$, the superiority of the power multivector is prominent compared to the clasical Budeanu's power equation. The power multivector can be compensated in various directions denoted by the basis bivectors. The case study compares $\tilde{S}$ to $\tilde{S}^{\prime}$ and $\tilde{\Omega}^{\wedge}$ to $\tilde{\Omega}^{\prime \wedge}$. This does not seem possible in the classical approaches, where power $\mathrm{S}$ (squared) is the sum of the magnitudes (squared) of the individual components of power multivector $\tilde{S}$.

- Finally, the most general method of power factor compensation for linear and non-linear loads in smart buildings is to add load compensating devices in order that the resultant load has a resistive behaviour (dissipasitivity condition). The application of these new concepts to the compensation theory should make significant improvements that deserve further research.

\section{CONCLUSIONS}

This work provides the power quality problems that exist in smart buildings. The consequence of electrical disturbance has become more serious considering the present economic situation. The massive application of compact fluorescent and led lamps in smart buildings could be worse than the computers as they produce harmonic pollution causing network voltage and current distortion. It was proved that power consumption figures would be significantly less at lower power factor, but more investigations into residential, commercial and industrial loads are needed to establish this. This paper advances a compensator design for power factor improvement based on dissipasitivity condition, from GA. The main contribution of this work is the proof that GA is a powerful tool to represent power and to handle the problem of direction and sense of different compensated components. This incorporates in a single expression all the required information for the minimization of each reactive power term by means of possible compensating devices, new optimization algorithms and effective power quality indices in smart buildings loads.

\section{REFERENCES}

[1] Recommended Practices and Requirements for Harmonic Control in Power Systems, IEEE Standard 519-92.

[2] Limits for Harmonic Current Emissions, IEC 61000, Part 3, Section 4, 1995.

[3] Steinmetz. C.P.. "Theory and calculation of alternating current phenomena". McGraw Publishing Company, New York.1908.Chaps. 15, 24 and 30.

[4] C. I. Budeanu, "Puissances reactives et Fictives", Instytut Romain de 1'Energie. Bucharest, Romania 1927.

[5] Fryze, S."Wik-Blind, un Scheinleitung in Elektrischen Stromkreisen mit nichtsinusoidalem Verlauf von Strom und Spanung " Elekt. TZ., vol.53,pp.596-599,625-627,700-702,1932.

[6] L.S. Czarnecki, "Budeanu and Fryze: Two frameworks for interpreting power properties of circuits with non sinusoidal voltages and currents". Archv. Elektrotechn., vol.80, no.6 pp. 359-367, 1997.

[7] L. S. Czarnecki, "Considerations on the reactive power in non-sinusoidal situations" IEE Trans. 1985, IM-34, pp. 399-404.

[8] L.S. Czarnecki, "What is wrong with thre Budeanu concept of reactive power and distortion power and why it should be rejected". IEEE Trans. Instrum. Meas. vo. IM-36, pp.834-837, 1987.

[9] W. Shepherd and P. Zhakikhani. " Suggested definition of reactive power for nonsinusoidal systems”. Proc. Inst.Elect. Eng. Vol.119,pp.1361-1362, Sep.1972.

[10] D. Sharon. "Reactive power definitions and power factor improvement in nonlinear systems". Proc IEE, Vol.120, Nº6, June 1973.

[11] M.A Slonim and J.D. Van Wyk. "Power components in a system with sinusoidal and nonsinusoidal voltages and/or currents" Proc. Inst. Elect Eng, Pt. B, vol.135,pp.76-84,Mar. 1988.

[12] Petroianu A.I.: "A geometric algebra reformulation and interpretation of Steinmetz's symbolic method and his power expression in alternating current electrical circuits", Electr. Eng., 2015, 97, pp. 175-180

[13] Petroianu A.I.: "Mathematical representations of electrical power: vector or complex number? neither!". Electrical Power and Energy Conf., Calgary, AB., November 2014, pp. 170-177.

[14] Chappell J.M., Drake S.P., Seidel C.L., ET AL.: 'Geometric algebra for electrical and electronic engineers', Proc. IEEE, 2014, 102, (9), pp. 1340-1363

[15] A.M. Sommariva, “ Power Analysis of One-Ports Under Periodic MultiSinusoidal Operation”, IEEE Trans. On Circuits and Systems.-I: Regular Papers, Vol.53.Nº. September 2006.

[16] M. Castilla, J.C. Bravo, M. Ordoñez, J.C. Montaño: "Clifford Theory: A Geometrical Interpretation of Multivectorial Apparent Power". IEEE Transactions On Circuit and Systems I-Regular Papers, Vol.55, No.10, pp. 2008. November 2008.

[17] R. Ortega, E. Garcia-Canseco, R. Griñó, A. Stankovic: “A CicloDissipasitivity Condition for Power Factor Improvement in Electrical Circuits". Proceedings of the Amareican Control Conference,pp.24922497. Minneapolis, Minnesota, USA ,June 2006.

[18] M. Castilla, J.C. Bravo, M. Ordoñez: "Geometric Algebra: A Multivectorial Proof of Tellegen's Theorem in Multiterminal Networks". IET Circuits, Devices and Systems, Vol.2, No.4, August 2008.

[19] J. Enbling and G. Scheuermann: "Clifford Fourier Transform on Vector fields," IEEE Transactions on Visualization and Computer Graphics, Vol.11, Issue 4, pp.469-479, July-Aug. 2005.

[20] D. Hestenes, G. Sobczyk: "Clifford Algebra to Geometric Calculus: a unified language for Mathematics and Physics”. (Kluwer Academic, Dordrech/Boston 1986). 